\title{
Enabling Accessible Interfaces to Digital Library Content
}

\author{
Syed Toufeeq Ahmed, K. Selçuk Candan, Suganthi Cidambaram, Shruti Gaur, Jong Wook Kim, \\ Mijung Kim, Hari Sundaram, Xinxin Wang and Renwei Yu \\ Dept. of Computer Science and Engineering \\ Arizona State University \\ Tempe, Arizona-85287 \\ Toufeeq@asu.edu
}

\begin{abstract}
Most of the web interfaces are primarily designed for people with sight, with visually rich features that makes effective use of the tools to enhance visual usability but in process making it impossible for users who are blind or visually impaired to use them. In this work, our goal is to improve participation to NSF's National Science Digital Library ${ }^{1}$ (NSDL) by teachers, librarians, and learners who are blind. The middleware for accessible information spaces on NSDL (MAISON) is enhancing the accessibility of NSDL, its internal and external resources and existing services (such as strand maps of educational benchmarks). Relying on cutting-edge, context-aware graph segmentation, filtering and summarization, and concept propagation techniques, the middleware provides information space adaptation, reduction, and preview services through open web-based service APIs to enable implementation of informative navigation interfaces that are able to reduce the complexity of the information space and provide previews to prevent user disorientation.
\end{abstract}

Keywords- Digital library navigation aids; assistive technology for blind users; educational content and web sites; summarization; annotation;

\section{INTRODUCTION}

Interfaces used by individuals who are blind mainly rely on presenting information in a linear, single-resolution fashion that allows very little room for communicating spatial dependencies between individual interface elements. Persons who are blind can access the information on the web generally only via a screen reader program (JAWS for Windows), which provides output in audio and/or Braille format. Even though screen reader software is helpful, it has limitations. It is generally very difficult, as well as time consuming, to find a link of interest, or a piece of information on a web page using a screen reader program, since the user has to listen to the screen reader speaking out each link as well as textual content, and must rely solely on the keyboard access because the use of a mouse is not practical without sight.

\section{RELATED WORK}

The AnnotatEd system, [2] integrated web page annotations and adaptive navigation techniques to support browsing-based access to information. Nonetheless, these approaches are designed to help sighted individuals, thereby not offering much support for our target group of users who are blind, as visual cues are not applicable, and auditory cues are costly in terms of cognition. In [3] we introduced OASIS which is a fully accessible digital library to support students in who are blind or visually impaired in successfully accessing books and other educational materials.

\section{MidDlEWARE FOR ACCESSIBLE INFORMATION SPACES}

MAISON is implemented as a middleware service to help minimize the extraneous load on users who are blind, while they search and access to materials from the large and diverse collection of resources available on NSDL. MAISON establishes as precisely as possible, the information needed, based on the current search context and user preferences. It also attempts to keep the presentation related intrinsic overload to a minimum by selecting contextually appropriate presentation modalities (Figure 1(a)). NSDL's original strand map service (SMS) can be accessed through a Concept Space Interchange Protocol (CSIP) which is a REpresentational State Transfer (REST) service API that lets developers search through concept maps and find digital library resource supporting concept maps. MAISON uses the CSIP API [1] to query and access StrandMaps and adapt them based on user provided context and adaptation strategies (Figure 1(c)). In particular, each CSIP request returns one or more strand map nodes that satisfy the user query and their connections. Our CSIPAccessible (CSIP-A) interface, however, takes (in addition to a set of search keywords) various adaptation options that are used for adapting the CSIP strand map results based on the user's preferences. These adaptation options include

- Grade/topic focus: This tells the MAISON middleware whether the adaptation should be grade- (or row-) centric or topic- (or column-) centric.

- Clustering: If this option is yes, related benchmark nodes of the strand map maybe clustered together to reduce the number of nodes to be presented and the links that has to be followed by the user who is blind.

- Link preview: This link previews are annotations for helping the user who is blind to decide whether to follow a forward or backward link. There are three alternatives:

1. Content of the next node option provides the content of the next node;

${ }^{1}$ http://nsdl.org 


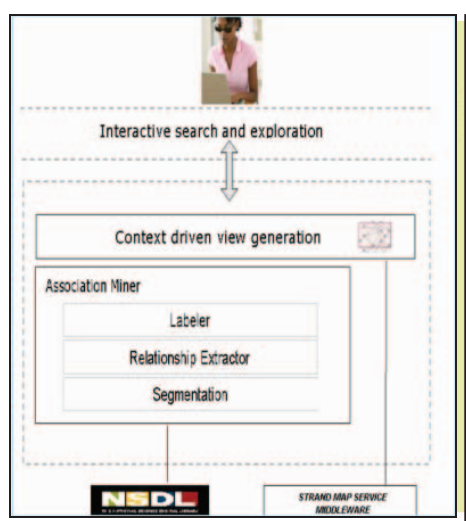

(a)

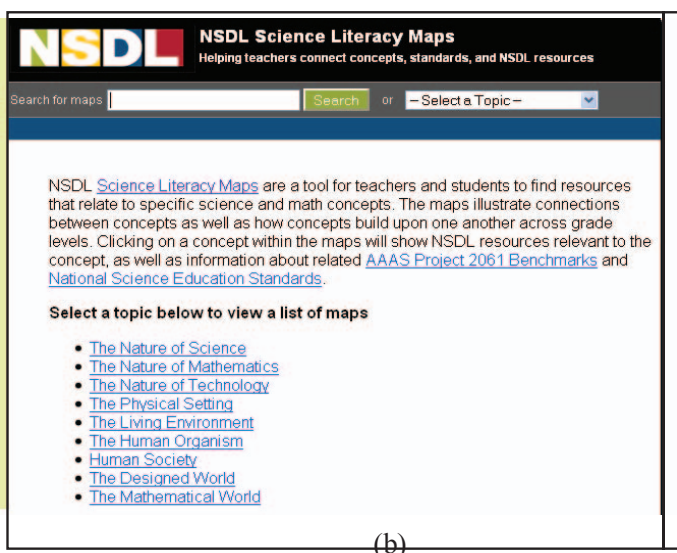

(b)

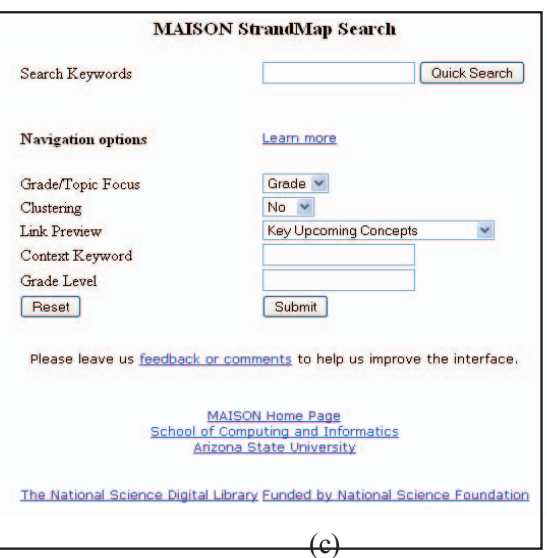

(c)

Figure 1 (a) MAISON architectural overview; (b) The original NSDL search interface; and (c) MAISON search interface (In addition to search keywords, the MAISON interface provide mechanisms for the user to describe adaptation and navigation strategies)

2. Upcoming Key concepts option identifies and annotates each edge on the resulting strand map with concepts that are dominant in upcoming (one or more) benchmarks (e.g. Figure 2);

3. Summary of the upcoming nodes option extracts a textual summary of the upcoming (one or more) benchmarks;

- Context keywords: Different from the search keywords, these keywords do not decide which benchmarks will be included in the result. Instead, they affect benchmark clustering, concept extraction and propagation, and textual summary generation schemes.

- Grade Level: This option helps the user who is blind focus the adaptation to a particular grade level.

The middleware provides a new CSIP-A protocol (Figure 3) that enables development of user interfaces that can deliver contextually informed information space adaptation. The adaptation strategies currently implemented include information space reduction, upcoming key concepts preview, and neighborhood summary preview. The ongoing extensions to the MAISON middleware include document, web page, and web site adaptation and preview APIs for improved access to external digital resources link to the underlying information space.

\section{REFERENCES}

[1] T. Sumner, F. Ahmad, Q. Gu, F. Molina, S. Willard, M. Wright, L. Davis, S. Bhushan and G. Janee, A Web Service Interface for Creating Concept Browsing Interfaces, D-Lib Magazine, 10 (2004), pp. 10829873.

[2] Farzan, R. and Brusilovsky, P. 2008. AnnotatEd: A social navigation and annotation service for web-based educational resources. New Rev. Hypermedia Multimedia 14, 1 (Jan. 2008), 3-32.

[3] Q. Li, K. S. Candan, M. Goveas, S. Han, Terri Hedgpeth, J. W. Kim, A. Kolhatkar and M. L. Sapino, OASIS System for Organizing, Annotating, and Serving Information to Students without Sight, accepted for publication in the First International Conference on Technology-based Learning with Disability, 2007.

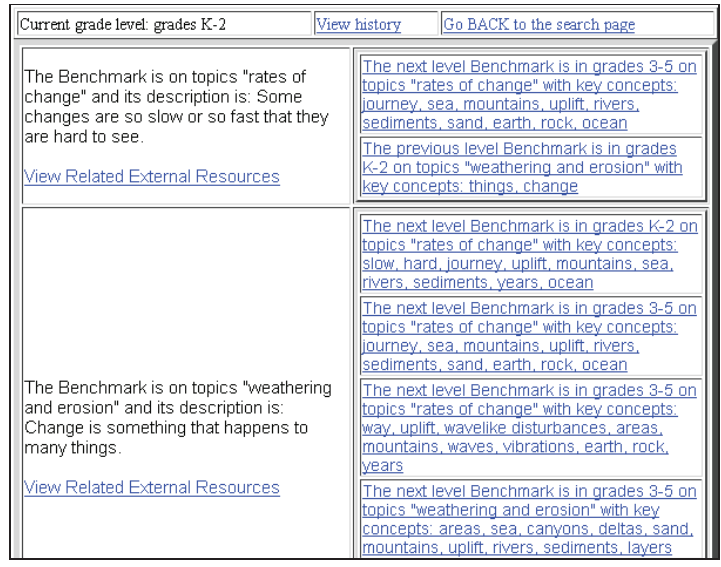

Figure 2. MAISON navigation interface: upcoming key concepts visualization

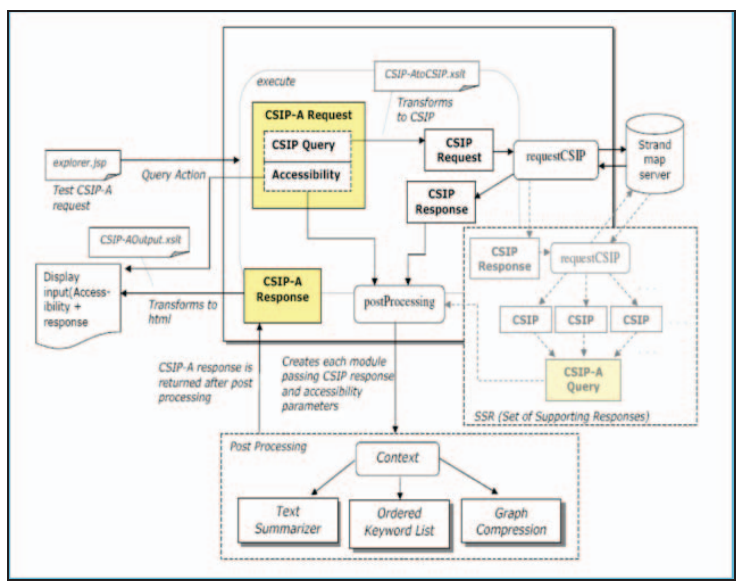

Figure 3 MAISON CSIP-Accessible (CSIP-A) Architecture.

This work is supported by NSF Award \#0735014, "MAISON: Middleware for Accessible Information Spaces on NSDL". 